Background The vulnerability to infection observed in newborns is associated with a limited ability to mount efficient immune responses. Elevated circulating levels of anti-inflammatory mediators (adenosine, prostaglandins,...) reduce production of proinflammatory cytokines by newborn monocytes upon microbial challenge. We hypothesised that macrophage migration inhibitory factor (MIF), a pro-inflammatory cytokine constitutively expressed in blood and immune cells, counter-balances the antiinflammatory milieu of newborns.

Methods MIF plasma levels were measured by ELISA in 200 healthy subjects, from birth to adulthood. Cord blood monocytes from term newborns were transfected with MIF siRNA, or were incubated with the MIF antagonist ISO-1, recombinant MIF, adenosine and prostaglandin E2 (PGE2) and then stimulated with endotoxin, bacterial lipopeptide, Escherichia coli and Group B Streptococcus (GBS). Intracellular proteins and cell-culture supernatants were collected to quantify MAPK phosphorylation by Western blot and cytokines by ELISA/Luminex.

Results Circulating MIF levels were 10-fold higher in newborns than adults, and decreased during infancy. Newborn monocytes expressed high MIF levels, and released MIF upon stimulation with Escherichia coli and GBS. Inhibition of MIF expression with MIF siRNA or MIF activity with ISO-1 reduced 1.5-5.7fold microbial product-induced secretion of pro-inflammatory (TNF, IL-1 $\beta$, IL-6, IL-8, IL-12p40, IL-12p70, IL-23) and antiinflammatory (IL-10, IL-20, IL-27) cytokines and phosphorylation of p38 and ERK1/2 MAPKs. Recombinant MIF counterregulated adenosine and PGE2-mediated inhibition of TNF production in Escherichia coli-stimulated newborn monocytes.

Conclusions MIF expression is developmentally regulated, with strikingly elevated levels in newborns compared to adults. High MIF levels at birth may act to balance pro/anti-inflammatory immune responses in newborns.

\section{Neonatal Resuscitation}

\section{0-066 ADMISSION HYPOTHERMIA IN VERY PRETERM INFANTS IS ASSOCIATED WITH MORTALITY - RESULTS FROM THE EPICE COHORT}

${ }^{1} \mathrm{E}$ Wilson, ${ }^{2} \mathrm{RF}$ Maier, ${ }^{3} \mathrm{~B}$ Misselwitz, ${ }^{4} \mathrm{~J}$ Zeitlin, ${ }^{5}$ AK Bonamy. ${ }^{1}$ CLINTEC, Karolinska Institutet, Stockholm, Sweden; ${ }^{2}$ Pediatrics, Philipps University, Marburg, Germany; ${ }^{3}$ Quality Assurance Hesse, Institute of Quality Assurance Hesse, Eschborn, Germany; ${ }^{4}$ Obstetrical Perinatal and Pediatric Epidemiology Research Team Center for Epidemiology and Biostatistics, INSERM, Paris, France; ${ }^{5}$ Women's and Children's Health Clinical Epidemiology Unit Department of Medicine Solna, Karolinska Institutet, Stockholm, Sweden

\subsection{6/archdischild-2014-307384.134}

Background Strategies to prevent heat loss in the delivery room after very preterm birth have been proven effective in randomised controlled trials. Nevertheless, we hypothesise that hypothermia at admission to neonatal care is still common and contributes to mortality after very preterm birth.

Methods The EPICE cohort included all births between $22+0$ and $31+6$ weeks of gestation in 19 regions from 11 European countries in 2011-2012. We studied infants surviving to admission to neonatal care ( $\mathrm{n}=7577)$. The association between temperature at admission and in-hospital mortality was analysed using logistic regression. The final model adjusted for gestational age, small for gestational age (SGA), Apgarscore $<7$ at $5 \mathrm{~min}$, infant sex and region of birth.

Results Of 6639 infants with data on body temperature at admission, 1670 infants $(25 \%)$ were hypothermic $\left(<36.0^{\circ} \mathrm{C}\right)$; $6 \%$ had temperatures $<35^{\circ} \mathrm{C}, 7 \%$ between 35.0 and $35.4^{\circ}$ and $12 \%$ between 35.5 and $35.9^{\circ}$. Body temperature at admission was inversely related to mortality. The crude odds ratio (OR) (95\% confidence interval [CI]) for mortality was 5.81(4.277.92) when temperature was $<35^{\circ} \mathrm{C} ; 3.32(2.35-4.69)$ at $35.0-$ $35.4^{\circ}$; and $1.61(1.18-2.19)$ at $35.5-35.9^{\circ}$ compared to normothermic infants $\left(36.5-37.5^{\circ} \mathrm{C}\right)$. After adjustment, temperatures below $35.5^{\circ} \mathrm{C}$ remained significantly associated with mortality, $1.94(1.32-2.83)$ at $<35^{\circ} \mathrm{C}$ and $1.91(1.30-2.82)$ at $35-35.4^{\circ} \mathrm{C}$ compared to normothermic infants.

Conclusion Hypothermia after very preterm birth contributes to mortality in modern perinatal care settings in Europe. Further studies should investigate if evidence-based heat loss prevention strategies have been implemented.

\section{0-067 THE INFLUENCE OF LOW QUALITY PULSE OXIMETRY SIGNALS ON EVALUATING INFANTS AT BIRTH}

${ }^{1} \mathrm{IC}$ Narayen, ${ }^{2} \mathrm{M}$ Smit, ${ }^{3} \mathrm{EW}$ van Zwet, ${ }^{4} \mathrm{JA}$ Dawson, ${ }^{5} \mathrm{NA}$ Blom, ${ }^{1} \mathrm{AB}$ te Pas. ${ }^{1}$ Neonatology, Leiden University Medical Center, Leiden, Netherlands; ${ }^{2}$ Obstetrics, Leiden University Medical Center, Leiden, Netherlands; ${ }^{3}$ Medical Statistics, Leiden University Medical Center, Leiden, Netherlands; ${ }^{4}$ Neonatal Services, The Royal Women's Hospital, Melbourne, Australia; ${ }^{5}$ Pediatric Cardiology, Leiden University Medical Center, Leiden, Netherlands

\subsection{6/archdischild-2014-307384.135}

Objectives Reference ranges for pulse oximetry (PO) measurements of oxygen saturation $\left(\mathrm{SpO}_{2}\right)$ and heart rate (HR) in infants at birth were generated excluding measurements with system messages (SyM), such as 'Low Signal Quality' or 'Low perfusion'. However, in clinical practice SyM often occur and may not be noticed. To assess the usability of measurements obtained with SyM, we investigated the differences of $\mathrm{SpO}_{2}$ and $\mathrm{HR}$ between measurements with and without SyM and if reference ranges would change when including data with SyM.

Methods $\mathrm{HR}$ and $\mathrm{SpO}_{2}$ of uncompromised term infants were recorded using Masimo PO. Differences in mean and standard deviation (SD) between measurements with and without SyM were calculated and compared with permutation tests. Centile charts were computed with and without SyM.

Results PO values of 138 neonates were obtained, resulting in 28,477 data points. SyM occurred in $46 \%$ of measurements. Most SyM occurred in the 3rd and 4th minute after birth. Mean $\mathrm{SpO}_{2}$ with SyM was lower $(\mathrm{p}<0.001)$, SD was similar to data without SyM. SyM included, centile charts of $\mathrm{SpO}_{2}$ are approximately 3\% lower, but not more dispersed. Mean HR was lower $(\mathrm{p}<0.001)$ and more dispersed $(\mathrm{p}<0.001)$ when SyM occurred. SyM included centile charts of HR have increased variability and are lower.

Conclusion In term infants at birth SyM on PO occurred frequently. $\mathrm{SpO}_{2}$ measurements with $\mathrm{SyM}$ are reliable for monitoring an infant's clinical condition. However, HR measurements with SyM should be used with caution as they may underestimate an infant's HR. 